\title{
Determinants of Visceral Infarction in Acute Cardioembolic Stroke Due to Atrial Fibrillation
}

\author{
Sung-Il Sohn, ${ }^{\mathrm{a}, *}$ Sang-Won Park, ${ }^{\mathrm{b}, *}$ Younghyurk Lee, ${ }^{\mathrm{a}}$ Hyungjong Park, ${ }^{\mathrm{a}}$ Hyukwon Chang, ${ }^{\mathrm{c}}$ H. Alex Choi, ${ }^{\mathrm{d}}$ \\ Jeong-Ho Hong ${ }^{\mathrm{a}}$ \\ aDepartment of Neurology, Keimyung University Dongsan Hospital, Keimyung University School of Medicine, Daegu, Korea \\ ${ }^{b}$ Department of Neurology, Daegu Fatima Hospital, Daegu, Korea \\ 'Department of Radiology, Semyung Radiology Clinic, Gumi, Korea \\ ${ }^{d}$ Department of Neurology and Neurosurgery, McGovern Medical School, University of Texas Health Science Center at Houston, Houston, TX, USA
}

*These authors contributed equally to the manuscript as first author.

\section{Dear Sir:}

Atrial fibrillation (AF) is associated with an increased risk of systemic embolism and cardioembolic stroke. However, research on coexisting subdiaphragmatic visceral infarctions (SDVIs) with acute ischemic stroke due to AF is scarce. The most frequent localization of SDVI is the kidneys. ${ }^{1}$ Acute kidney injury following acute renal infarction can occur in $20 \%$ to $40 \%$ cases, and chronic kidney injury, in about $34 \%$ of cases. ${ }^{2}$ Splenic infarction was the second most common SDVI in AF-related acute ischemic stroke, with a $5 \%$ mortality rate. ${ }^{3}$ Acute mesenteric ischemia is an infrequent event, though with high mortality risk. ${ }^{4}$ SDVI diagnosis is frequently missed or delayed because of nonspecific clinical symptoms. Therefore, it is essential to predict coexisting acute SDVI in AF-related stroke patients.

Patients with acute cardioembolic stroke due to nonvalvular AF within 7 days of the onset of symptoms were prospectively included at two tertiary hospitals between April 2016 and February 2018. All subjects were examined using abdominal magnetic resonance imaging (MRI) and transthoracic echocardiography (TTE) within 7 days of onset. Detailed criteria of subjects and MRI protocols were described in the Supplementary methods $^{5}$ and Supplementary Figure 1.

A total of 100 consecutive nonvalvular AF-related acute cardioembolic stroke patients were enrolled. The median age was 74 years (interquartile range, 68 to 79), and 47\% were male. Among those patients, acute and chronic SDVls occurred in 31
(31\%) patients. Comparisons of baseline characteristics between each group are shown in Table 1. One patient had acute and chronic SDVI simultaneously. Twenty-three acute coexisting SVDls were found in 20 patients (20\%): 20 cases of renal infarction (Supplementary Figure 2), two splenic infarction, and one mesenteric infarction due to superior mesenteric artery occlusion. Of the 20 patients with acute renal infarction, four had an acute infarction in both kidneys. Furthermore, 12 patients had previous chronic SDVIs (10 with renal, two splenic infarction). One patient had chronic renal infarction on both sides. No hepatic infarction was shown (Supplementary Table 1). In 96 patients with available TTE data, the rate of moderate to severe left atrial enlargement (LAE), defined as volume/body surface area $\geq 42 \mathrm{~mL} / \mathrm{m}^{2}$, was $69.8 \%$.

In the multiple logistic regression model, moderate to severe LAE was significantly associated with the coexistence of acute SDVIs in patients with acute ischemic stroke due to AF (adjusted odds ratio $[\mathrm{aOR}], 11.40 ; 95 \%$ confidence interval $[\mathrm{Cl}], 1.29$ to 100.97; $P=0.03$ ), and also a significant determinant for the presence of all SDVIs stages $(\mathrm{aOR}, 5.12 ; 95 \% \mathrm{Cl}, 1.37$ to 19.15 ; $P=0.02$ ) (Table 2).

Our study found that 31\% of patients with acute cardioembolic stroke attributed to AF, presenting within 7 days of onset, showed some stage of SVDI. Acute coexisting SVDIs occurred in $20 \%$ of total cases and chronic SDVIs in $12 \%$. In one postmortem study, SDVI was observed in $38.6 \%$ of patients with cardioembolic stroke. ${ }^{1}$ However, this study had a potential for selection bias (only fatal strokes were included), and it could 


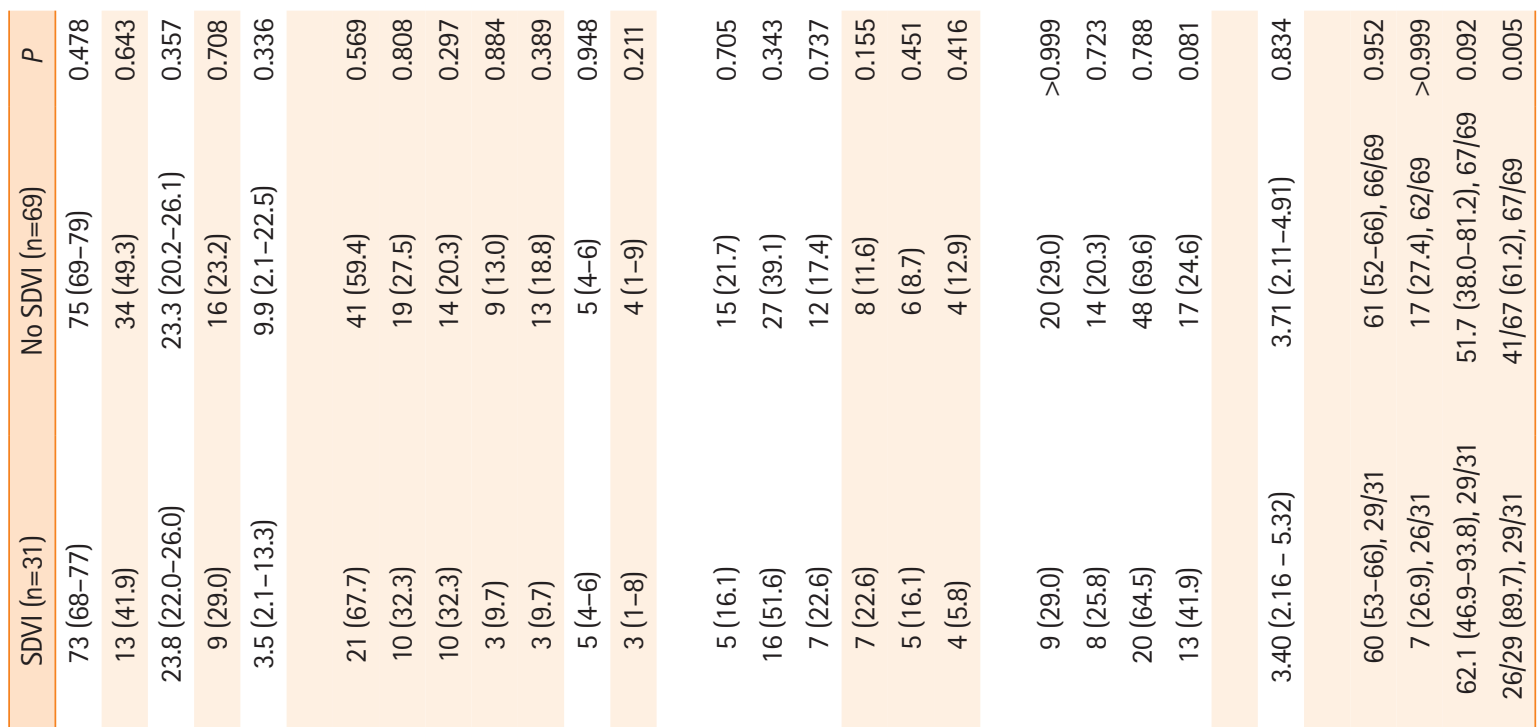

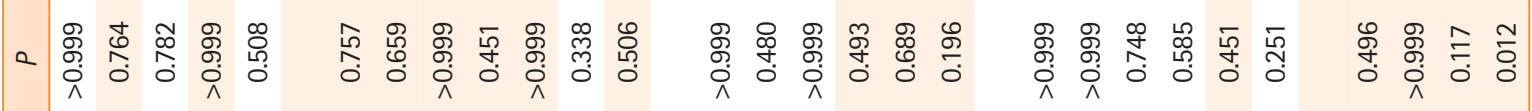

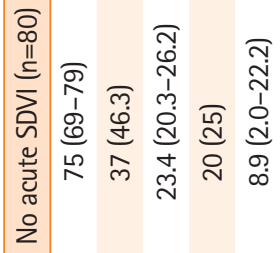

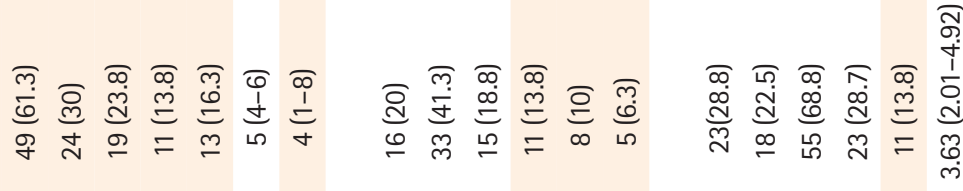
$\stackrel{\infty}{\infty}$

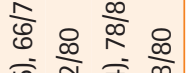

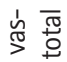
莀

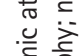
हुำ 는 흥 똔 원 产 $\frac{\pi}{\overline{0}}$ 虎 嵌 苋. 늘 $\stackrel{\frac{\pi}{d}}{\frac{\pi}{\pi}}$ 
Table 2. Independent predictors associated with subdiaphragmatic visceral infarction

\begin{tabular}{lcc}
\hline Variable & OR & $95 \% \mathrm{Cl}$ \\
\hline Acute subdiaphragmatic visceral infarction & & $P$ \\
Age (yr) & 0.999 & $0.941-1.061$ \\
Male sex & 0.806 & $0.248-2.615$ \\
Endovascular treatment & 1.874 & $0.363-9.681$ \\
Moderate to severe LAE & 11.401 & $1.287-100.965$ \\
Onset-to-abdominal MRI time (day) & 1.272 & $0.965-1.676$ \\
Acute and chronic subdiaphragmatic visceral infarction & & 0.454 \\
Age (yr) & 0.999 & 0.029 \\
Male sex & 1.270 & $0.945-1.055$ \\
Initial NIHSS score & 0.965 & $0.468-3.446$ \\
Posterior circulation lesion & 2.036 & $0.885-1.052$ \\
Acute treatment & 2.275 & $0.728-5.691$ \\
Moderate to severe LAE & 5.123 & 0.964 \\
\hline
\end{tabular}

$\mathrm{OR}$, odds ratio; $\mathrm{Cl}$, confidence interval; $\mathrm{LAE}$, left atrial enlargement; $\mathrm{MRI}$, magnetic resonance imaging; NIHSS, National Institutes of Health Stroke Scale.

not assess the temporal link between acute cardioembolic stroke due to AF and concomitant acute SDVIs.

Abdominal MRI, including diffusion-weighted imaging, can easily differentiate between recent and old infarctions, and we performed an abdominal MRI within 7 days of symptom onset. Furthermore, there is no additional harm to patients suspected of accompanying acute renal infarction due to no need to use contrast agents compared to abdominal computed tomography. However, there is a limit to the implementation in patients with unstable vital signs. In a small MRI-based study, four (14.8\%) out of 27 stroke patients with AF showed recent infarctions and three (11.1\%) old infarctions. ${ }^{6}$ Another MRIbased study found SDVIs in 10 (21.3\%) out of 47 patients with ischemic stroke due to AF. ' Of those, 10.7\% had acute and $10.6 \%$ chronic infarctions. In terms of acute SDVIs, our study showed a higher incidence than previous studies. This finding may be due to a higher rate of vascular comorbidities and a higher risk of the $\mathrm{CHA}_{2} \mathrm{DS}_{2}$-VASc score in our subjects. The prevalence of chronic SDVIs was similar to previous MRI-based studies.

Our results suggest that a moderate-to-severe LAE on TTE is more closely related to a greater risk of acute coexisting SDVIs, in acute cardioembolic stroke due to $A F$, compared to normal left atrium (LA) or mild $L A E$, which were independent of the SDVI stage. Atrial remodeling and impaired atrial contractility may contribute to $L A E$, which in turn may lead to more blood stasis and endothelial injury, thereby predisposing to thromboembolism. Thrombus formation is more likely to occur in the larger LA. Large LA volume could be a good predictor of an increased risk of ischemic stroke, systemic embolism, and mor- tality in AF patients without a history of previous stroke. ${ }^{8}$ The association between LAE and recurrent stroke is already well known. However, before our study, the association between LAE and systemic embolism in AF-related acute cardioembolic stroke was less clear. Based on our results, evaluating the coexistence of acute SDVIs is critical in AF-related acute cardioembolic stroke patients with moderate-to-severe LAE.

The interpretation of our results require caution because of selection bias. In contrast to the autopsy study, some patients with unstable vital signs or malignant stroke may have been excluded from the study. Non-categorization of AF type is also considered an important limitation. However, LA volume is positively correlated with AF duration and could be a good marker of AF duration.

\section{Supplementary materials}

Supplementary materials related to this article can be found online at https://doi.org/10.5853/jos.2020.03972.

\section{References}

1. Abboud H, Labreuche J, Gongora-Riverra F, Jaramillo A, Duyckaerts $C$, Steg PG, et al. Prevalence and determinants of subdiaphragmatic visceral infarction in patients with fatal stroke. Stroke 2007;38:1442-1446.

2. Kwon JH, Oh BJ, Ha SO, Kim DY, Do HH. Renal complications in patients with renal infarction: prevalence and risk factors. Kidney Blood Press Res 2016;41:865-872.

3. Nores M, Phillips EH, Morgenstern L, Hiatt JR. The clinical 
spectrum of splenic infarction. Am Surg 1998;64:182-188.

4. Schoots IG, Koffeman GI, Legemate DA, Levi M, van Gulik TM. Systematic review of survival after acute mesenteric ischaemia according to disease aetiology. Br J Surg 2004;91:17-27.

5. Ko Y, Lee S, Chung JW, Han MK, Park JM, Kang K, et al. MRIbased algorithm for acute ischemic stroke subtype classification. J Stroke 2014;16:161-172.

6. Slaoui T, Klein IF, Guidoux C, Cabrejo L, Meseguer E, Abboud $\mathrm{H}$, et al. Prevalence of subdiaphragmatic visceral infarction in cardioembolic stroke. Neurology 2010;74:1030-1032.

7. Weisenburger-Lile D, Lopez D, Russel S, Kahn JE, Veiga Hellmann A, Scherrer A, et al. IRMA study: prevalence of subdiaphragmatic visceral infarction in ischemic stroke and atrial fibrillation. Int J Stroke 2017;12:421-424.

8. Hamatani Y, Ogawa H, Takabayashi K, Yamashita Y, Takagi D,
Esato $M$, et al. Left atrial enlargement is an independent predictor of stroke and systemic embolism in patients with non-valvular atrial fibrillation. Sci Rep 2016;6:31042.

Correspondence: Jeong-Ho Hong

Department of Neurology, Keimyung University Dongsan Hospital, Keimyung University School of Medicine, 1035 Dalgubeol-daero, Dalseo-gu, Daegu 42601, Korea

Tel: +82-53-258-7839

Fax: +82-53-258-4380

E-mail: neurohong79@gmail.com

https://orcid.org/0000-0002-8235-9855

Received: September 20, 2020

Revised: December 21, 2020

Accepted: December 28, 2020

The authors have no financial conflicts of interest. 


\section{Supplementary methods}

\section{Ethics statement}

Local Institutional Review Boards (IRBs) approved the collection of anonymized clinical data with patients' consent into the registry database to monitor and improve the quality of stroke care (IRB approval No. 2015-12-027 and DFH16MR00289).

\section{Study population}

Patients with acute cardioembolic stroke, due to nonvalvular atrial fibrillation (AF), who visited an emergency room within 7 days of symptom onset were prospectively included at two tertiary hospitals between April 2016 and February 2018. Magnetic resonance angiography (MRA) of the brain and neck was performed to exclude patients with significant stenosis of the middle cerebral and carotid arteries on the lesion side. AF was diagnosed based on medical history and based on standard or continuous electrocardiography during stroke unit admission. Therefore, AF was not classified according to type (paroxysmal, persistent, or permanent) as defined by episode timing and termination. According to the Trial of Org 10172 in Acute Stroke Treatment classification, using a magnetic resonance imaging (MRI)-based diagnostic algorithm, other etiologies affecting embolic stroke such as malignancies, severe infection, major bleedings, and two or more undetermined causes (coexistence of other stroke etiologies such as large artery atherosclerosis, small vessel occlusion, and cardioembolism) were also excluded. ${ }^{5}$ Patients with unstable vital signs and patients who did not consent to this study were also excluded. All patients were examined using abdominal MRI and transthoracic echocardiography within 7 days of onset. Therefore, patients who were first diagnosed with AF a week after symptom onset with 24-hour Holter monitoring and implantable devices were also excluded from our study. A detailed flow diagram showing study participant selection is shown in Supplementary Figure 1.

\section{Magnetic resonance imaging protocols}

MRI and MRA of the intra- and extracranial brain were performed using a 3.0 Tesla scanner. Brain diffusion-weighted imaging parameters (repetition time 7,500 ms, echo time [TE] 84 ms, matrix number $128 \times 128,2$ b values 0 and $1,000 \mathrm{~s} / \mathrm{mm}^{2}$ ) included a slice thickness measuring $5 \mathrm{~mm}$ and an inter-slice gap measuring $2 \mathrm{~mm}$. Prospectively, all patients underwent abdominal MRI at 1.5T (Magnetom Avanto, Siemens Medical Solutions, Erlangen, Germany) within 7 days of ictus. The following protocol was followed: the body coil served as a transmitter and a six-element spine matrix coil in combination with the body matrix as a receiver. After routine localization and T2weighted imaging, a series $\left(b=50,400,800 \mathrm{~s} / \mathrm{mm}^{2}\right)$ of isotropic diffusion-weighted imaging was acquired using a spin-echobased echo-planar imaging sequence in combination with spectral adiabatic inversion recovery fat suppression. The acquisition was gated using prospective acquisition correction (PACE) respiratory triggering (TR; 3,100 to 6,500 ms) and tuned with the following parameters: TE $71 \mathrm{~ms}$; slice thickness $6 \mathrm{~mm}$; slice gap $1.625 \mathrm{~mm}$; field of view $380 \times 285 \mathrm{~mm}$; matrix 192×115; bandwidth 1,736 Hz/pixel; averages 2; and Generalized Autocalibrating Partially Parallel Acquisitions (GRAPPA) factor 2. Diffusion gradients $(25 \mathrm{mT} / \mathrm{m})$ were applied in the phase, read, and Z-direction separately. In total, 32 transverse slices were acquired with a $1.625-\mathrm{mm}$ slice gap to cover the whole liver within an average total acquisition time of $8.1 \mathrm{~min}-$ utes (range, 4.7 to 11.1). The image acquisition took place in an interleaved mode; first slices 1, 4 and 7, 10, 13, 16, 22, 25, 28, 31 were consecutively acquired with $b=0$ value, then the same slices with $b=50$ value and so on up to $b=1,000$. Subsequently, slices 2,5 and $8,11,14,17,20,23,26,29,32$ were acquired in the same way, and finally slices 3,6 and 9, 12, 15, 18, 21, 24, 27,30 as well. A single board-certified radiologist blinded to the clinical information assessed the outcomes for acute and chronic subdiaphragmatic visceral infarctions (SDVIs).

\section{Statistical analyses}

To compare baseline characteristics according to the presence of acute SDVIs, the Pearson's chi-square test or Fisher's exact test was used for categorical variables and the Student's t-test or Mann-Whitney $\mathrm{U}$ test for continuous variables, when appropriate. A multivariable logistic regression analysis with predefined variables (age and sex) and a bivariate analysis $(P \leq 0.25)$ to assess potential confounders associated with SDVIs were performed to predict coexisting SDVI. Significance levels were set at $P<0.05$ for two-tailed tests. All statistical analyses were performed using SPSS version 19.0 (IBM Co., Armonk, NY, USA). 
Supplementary Table 1. Localization of subdiaphragmatic visceral infarction

\begin{tabular}{lcc}
\hline Variable & Acute SDVI $(n=20)$ & Chronic SDVI (n=12) \\
\hline Kidney & $20(100)$ & $10(83.3)$ \\
Spleen & $2(10)$ & $2(16.7)$ \\
Mesentery & $1(0.5)$ & $0(0)$ \\
\hline
\end{tabular}

Values are presented as number (\%). Acute and chronic SDVls occurred in 31 patients. One patient had an acute and chronic SDVI simultaneously. SDVI, subdiaphragmatic visceral infarction.

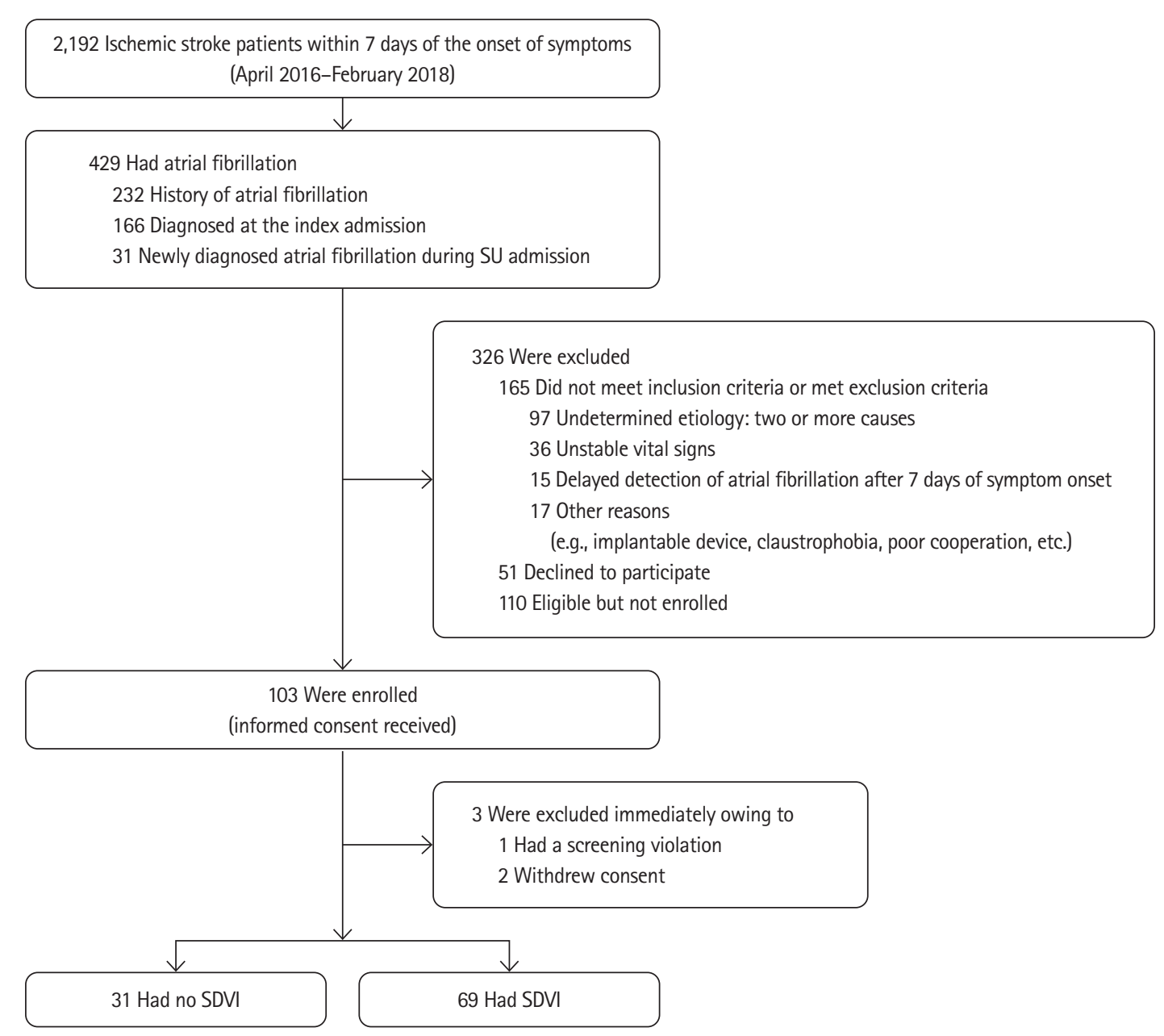

Supplementary Figure 1. Flow diagram of study participants. SU, stroke unit; SDVI, subdiaphragmatic visceral infarction. 

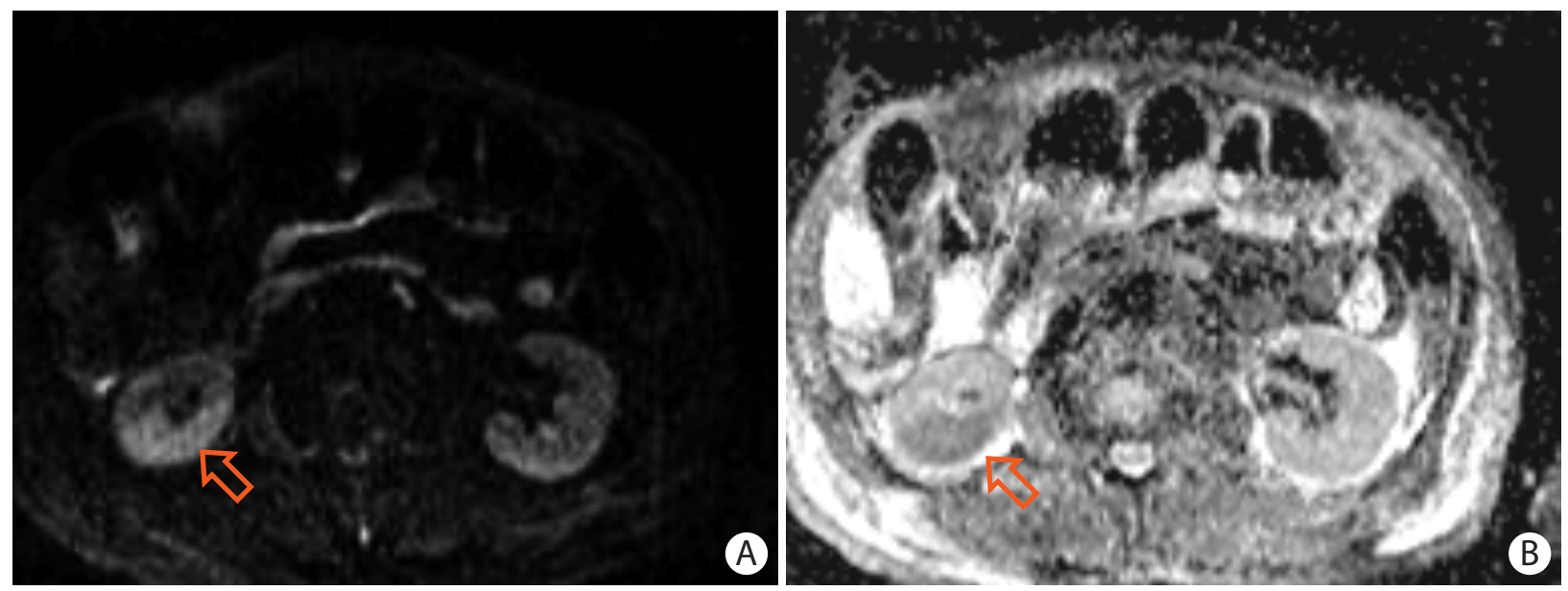

Supplementary Figure 2. Abdominal diffusion-weighted imaging (A) with the apparent diffusion coefficient (B) of 72-year-old patient, showing an area of acute renal infarction in the lower pole of the right kidney (arrows). 